\title{
Complications of first tarsometatarsal joint arthrodesis
}

\author{
Rodrigo Yuzo Masuda $^{(\mathbb{D})}$, Vinicius Felipe Pereiral ${ }^{(\mathbb{D}}$, Andre Vitor Kerber Cavalcante Lemos ${ }^{(\mathbb{D})}$, Caio Augusto de Souza Nery' ${ }^{(\mathbb{D})}$, \\ Nacime Salomão Barbachan Mansur ${ }^{1}$ (D) \\ 1. Universidade Federal de São Paulo - Escola Paulista de Medicina, São Paulo, SP, Brazil.
}

\begin{abstract}
Objective: First tarsometatarsal joint (TMTJ) arthrodesis, also known as Lapidus, is a surgical procedure used to treat severe hallux valgus, associated hypermobility of the first ray, and/or osteoarthritis of the first TMTJ. Despite the high satisfaction rate and high corrective power, this technique is not without complications. This study aimed to report the complications of first TMTJ arthrodesis.
\end{abstract}

Methods: This is a case series of 16 patients treated with first TMTJ arthrodesis. Patients were evaluated based on foot radiographs, clinical alignment of the hallux, and signs and symptoms.

Results: Eight patients had either major or minor complications. Three patients had recurrent deformity (1 with associated nonunion), 2 had delayed union, 2 had hardware loosening (1 with associated nonunion), and 1 had wound dehiscence.

Conclusion: First TMTJ arthrodesis requires greater care in choosing the surgical technique for the treatment of hallux valgus. In addition, some points should be considered to minimize complications as much as possible. We believe that data are still scarce to provide a concrete basis.

Level of Evidence IV; Therapeutic Studies; Case Series.

Keywords: Hallux valgus; Metatarsal bones; Tarsal bones; Pseudarthrosis; Arthrodesis.

\section{Introduction}

Hallux valgus, a term introduced by Carl Hueter(1) to define static subluxation of the first metatarsophalangeal joint characterized by lateral deviation of the hallux and medial deviation of the first metatarsal, ${ }^{(1,2)}$ is a disease for which different corrective surgical techniques can be used, depending on its severity. First tarsometatarsal joint (TMTJ) arthrodesis, also known as Lapidus, is one of the surgical procedures used in the treatment of severe hallux valgus, among other alterations ${ }^{(3-5)}$.

Despite the high satisfaction rate and high corrective power, this technique is not without complications. The main complications include delayed union, malunion, chronic edema, persistent pain, nonunion, joint stiffness, recurrent deformity, and varus deformity ${ }^{(6)}$. However, there is little scientific evidence of these complications after first TMTJ arthrodesis.

The purpose of this study was to report the complications of first TMTJ arthrodesis performed by orthopedic surgeons specializing in foot and ankle surgery.

\section{Methods}

This study was approved by the institutional research ethics committee and registered at Plataforma Brasil. We evaluated 16 patients undergoing first TMTJ arthrodesis, who denied any comorbidities. The procedures were performed by 7 different orthopedic surgeons specializing in foot and ankle surgery with different levels of experience, ranging from 1 to 29 years of experience in the field. All patients underwent arthrodesis with plates and screws. The plate was placed on the plantar aspect of the first tarsometatarsal in 4 patients and on the anteromedial aspect in all other patients. The dorsomedial incision was used, centered on the first TMTJ, for the 12 cases in which the plate was positioned in the dorsomedial region, and the medial incision was used for the 4 cases in which the plate was positioned on the plantar aspect. Also, articular cartilage was resected using a chisel and microperforations. All 16 patients were followed up in an outpatient clinic at 1, 2, $4,6,12$, and 24 weeks postoperatively. The follow-up visits included the evaluation of anteroposterior, lateral, and oblique
Study performed at the Universidade Federal de São Paulo - Escola Paulista de Medicina, São Paulo, SP, Brazil.

Correspondence: Rodrigo Yuzo Masuda. Rua Napoleão de Barros, 715 - Vila Clementino - 04024-002, São Paulo - SP, Brazil. E-mail: rodrigo.yu@gmail.com Conflicts of Interest: none. Source of funding: none. Date received: July 07 , 2021. Date accepted: November 23, 2021. Online: December 20, 2021.

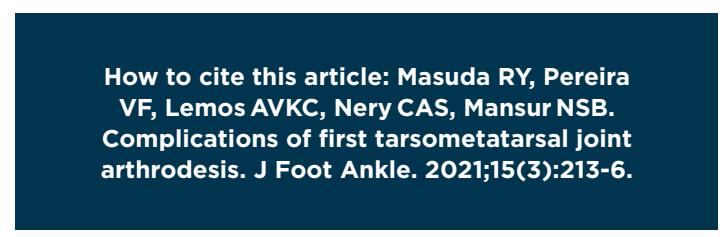


radiographs of the foot, clinical alignment of the hallux, and patients' signs and symptoms. For all patients, postoperative care included immobilization in a non-weight-bearing cast for 4 weeks, initiation of physical therapy with range of motion gain at 4 weeks, and partial weight-bearing at 6 weeks.

\section{Results}

We evaluated 16 patients operated on over an 18-month period (from February 2017 to August 2018). We measured preoperative and postoperative hallux valgus and intermetatarsal angles (Table 1). Eight patients had either major or minor complications (Table 2). Three patients had recurrent deformity ( 1 with associated nonunion), 2 had delayed union, 2 had hardware loosening (Figure 1) -1 with associated nonunion (Figure 2), and 1 had wound dehiscence.

\section{Discussion}

Surgical correction of hallux valgus using first TMTJ arthrodesis is technically difficult, requiring a steep learning curve

Table 1. Preoperative and postoperative hallux valgus angle (HVA) and intermetatarsal angle (IMA)

\section{Patient no. Preop HVA Postop HVA Preop IMA Postop IMA}

\begin{tabular}{llccc}
\hline 1 & 38 & 13 & 17 & 7 \\
\hline 2 & 40 & 12 & 17 & 6 \\
\hline 3 & 42 & 12 & 15 & 6 \\
\hline 4 & 43 & 14 & 14 & 5 \\
\hline 5 & 37 & 25 & 20 & 13 \\
\hline 6 & 35 & 15 & 22 & 10 \\
\hline 7 & 30 & 11 & 23 & 10 \\
\hline 8 & 39 & 29 & 17 & 14 \\
9 & 29 & 8 & 22 & 11 \\
10 & 27 & 7 & 22 & 9 \\
11 & 25 & 3 & 21 & 8 \\
12 & 29 & 10 & 19 & 5 \\
13 & 31 & 11 & 17 & 4 \\
14 & 31 & 12 & 17 & 10 \\
15 & 32 & 30 & 18 & 14 \\
16 & 26 & 5 & 16 & 10 \\
\hline
\end{tabular}

to perform the procedure ${ }^{(7)}$. Our literature review identified several articles describing the results and complications of this surgical technique, such as nonunion, delayed union, hardware-related discomfort, recurrent deformity, and loss of correction, among others ${ }^{(8,9)}$.

Nonunion was the most common complication in our study (25\%). Unlike our study, Mallette et al. ${ }^{(10)}$ reported a much lower rate of nonunion (8.3\%), as well as Espinosa et al. ${ }^{(6)}$ and Prissel et al. ${ }^{(11)}$, who reported lower rates of $16 \%$ and $6.5 \%$, respectively. In our study, patients who developed this complication underwent revision arthrodesis, with improved pain and stability after 1 year of outpatient follow-up.

Regarding hardware-related discomfort, Peterson et al. ${ }^{(12)}$ reported a rate of $15.2 \%$, thus requiring hardware removal. Of these, $72 \%$ had plates and $28 \%$ had crossed screws. This rate is also very different from that of our study (25\%). Our patients also required hardware removal to relieve pain resulting from hardware-related discomfort.

Regarding recurrent deformity, Schmid and Krause ${ }^{(13)}$ reported $16 \%$ of cases with this complication. In our study, we found a rate of $37.5 \%$, much higher than that reported in the literature, being the most common complication in our study. We defined recurrent deformity based on hallux valgus and intermetatarsal angles assessed on radiographs. At 24 weeks postoperatively, the 3 patients with this complication had a hallux valgus angle of $25^{\circ}, 29^{\circ}$, and $30^{\circ}$ and an intermetatarsal angle of $13^{\circ}, 14^{\circ}$, and $14^{\circ}$, respectively. Despite deformity recurrence, all 3 patients chose not to undergo another surgical procedure because they no longer had pain complaints.

Delayed union occurred as frequently as nonunion, with a rate of $25 \%$ in our study. Klos et al., ${ }^{(14)}$ however, reported a much lower rate of only $1.72 \%$. Our patients had pain complaints and no radiographic evidence of union until postoperative week 12. At 24 weeks, the patients had no pain complaints and showed evidence of union on radiographs; therefore, no additional procedure was performed.

In an attempt to avoid the most fearsome complication, nonunion, the literature describes several related factors that should be avoided or minimized. Barp et al. ${ }^{(15)}$ showed no statistically significant difference in the type of fixation used (plate vs screw). Blitz et al. ${ }^{(16)}$ reported that early weight-bea-

Table 2. Description of 8 complications of first tarsometatarsal joint arthrodesis

\begin{tabular}{lcllc}
\hline Patient no. & Age & Sex & Diagnosis & Complication \\
\hline 1 & 71 & Female & Hallux valgus & Hardware loosening + nonunion \\
\hline 2 & 55 & Female & Hallux valgus & Delayed union \\
3 & 63 & Female & Hallux valgus + hypermobility of the first ray & Wound dehiscence \\
\hline 4 & 40 & Female & Hallux valgus + hypermobility of the first ray & Hardware loosening \\
\hline 5 & 60 & Female & Hallux valgus + hypermobility of the first ray & Recurrent deformity \\
\hline 6 & 63 & Female & Hallux valgus + hypermobility of the first ray & Recurrent deformity + nonunion \\
\hline 8 & 75 & Female & Hallux valgus & Recurrent deformity \\
\hline
\end{tabular}


ring does not lead to higher nonunion rates. Moore et al. ${ }^{(17)}$ reported that patients with vitamin $D$ deficiency, in addition to endocrine diseases such as thyroid dysfunction and parathyroid disease, are 8.1 times more likely to develop nonunion, with a statistically significant difference compared with the control group.

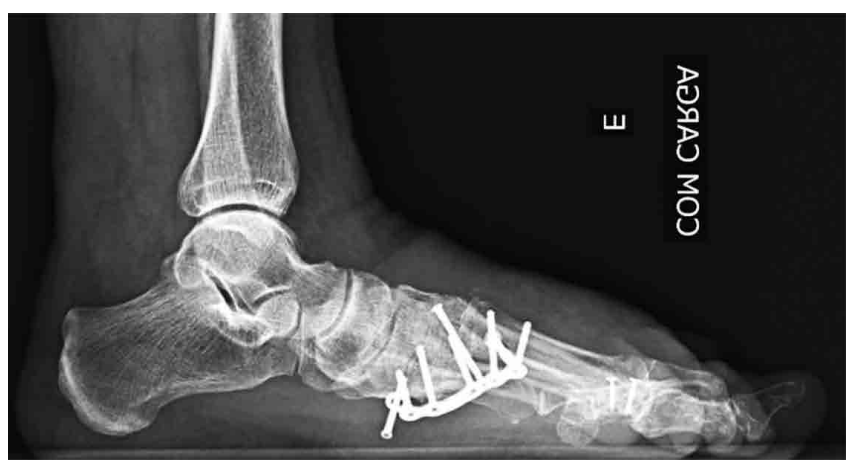

Figure 1. Radiographic evidence of hardware loosening in the left foot.

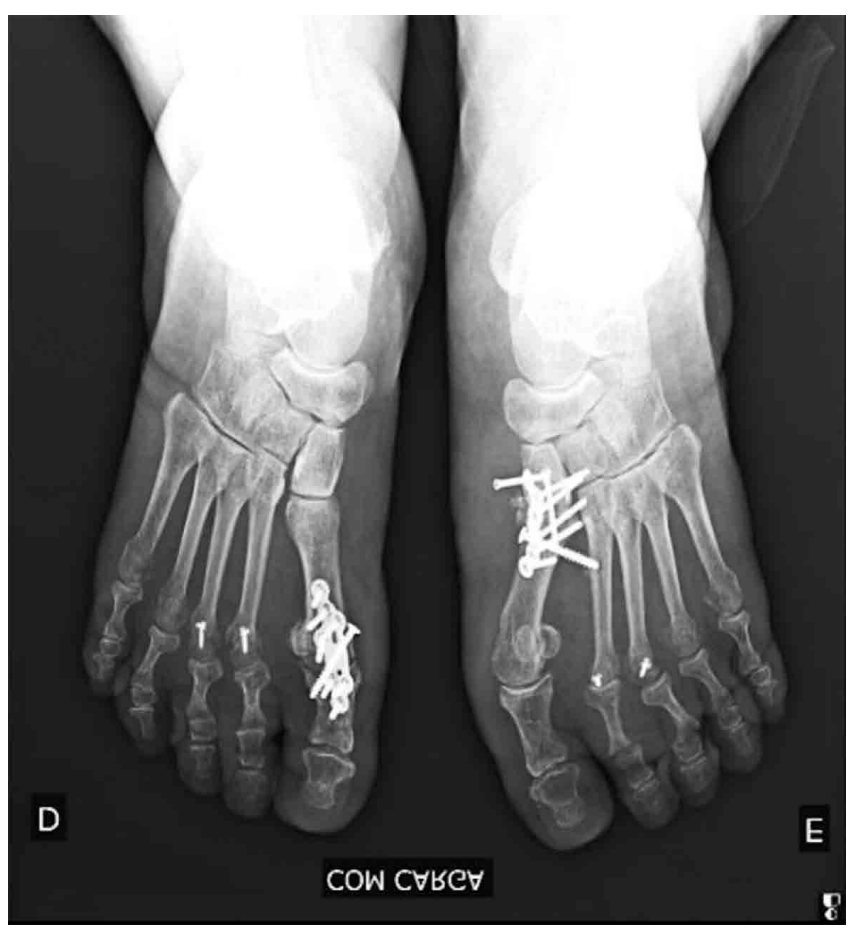

Figure 2. Radiographic evidence of hardware loosening in the left foot.
In order to reduce this complication, alternatives such as technique modifications and fragment rotation have produced encouraging results. Klemola et al. ${ }^{(18)}$ described a new first TMTJ arthrodesis technique by combining rotational correction of the first metatarsal and reported a $2 \%$ rate of nonunion. Simons et al. ${ }^{(19)}$ reported that first TMTJ arthrodesis with plantar plating resulted in lower rates of neurovascular injury. Klos et al. ${ }^{(14)}$ reported a rate of only $1.72 \%$ of nonunion in patients undergoing first TMTJ arthrodesis with a plate placed on the plantar aspect of the first TMTJ. Li and Myerson ${ }^{(20)}$ reported that some methods, such as plantar plating, nickel-titanium staples, and intramedullary fixation, produced greater biomechanical stability in cadaveric studies. In our study, 4 procedures were performed with plantar plating, and none of these patients developed nonunion. However, our small sample size prevents us from drawing conclusions that placing a plantar plate avoids this complication. Conti et al. ${ }^{(21)}$ reported a significant decrease in recurrence rates in the decreased first metatarsal pronation group compared with the no-change/increased first metatarsal pronation group.

The most relevant finding of our study is the difference in complication rates compared with those found in previous studies. We found a much higher rate for all complications. We believe that this difference is mainly due to the limited experience of some surgeons who performed the procedures, since, of 8 patients who developed complications, 6 had the procedure performed by a surgeon with only 2 years of experience. The limitations of this study include a small sample size, the lack of a control group, and the great difference in experience across surgeons, thus making it difficult to determine the true factors that contribute to the development of complications. Therefore, all the disagreements found between our complication rates and those reported in the literature indicate that further prospective studies are needed. The indication of a surgical procedure or surgical technique must be very well evaluated to avoid as much as possible the complications that can be minimized.

\section{Conclusion}

Based on our study, we can conclude that first TMTJ arthrodesis requires greater care in choosing the surgical technique for the treatment of hallux valgus. In addition, some points should be considered to avoid complications as much as possible, such as the use of implants to increase stability, instruments for better articular cartilage resection, and improvement in the correction of the pronation deformity of the hallux. The limitations of our study prevent us from drawing a robust conclusion and defining consistent parameters related to the main factors that may affect the various complications described in the study. Therefore, we believe that data are still scarce to provide a concrete basis, and further studies are needed to make substantiated conclusions. 
Authors' contributions: Each author contributed individually and significantly to the development of this article: RYM *(https://orcid.org/OOOO-00030414-5752) Conceived and planned the activities that led to the study, data collection, statistical analysis, wrote the article and approved the final version; VFP *(https://orcid.org/0000-0002-1005-6089) Conceived and planned the activities that led to the study, wrote the article, critical analysis and approved the final version; AVKCL *(https://orcid.org/0000-0001-8974-5815) Performed the surgeries, conceived and planned the activities that led to the study, critical analysis and approved the final version; CASN *(https://orcid.org/0000-0002-9286-1750) Performed the surgeries, conceived and planned the activities that led to the study, critical analysis and approved the final version; NSBM *(https://orcid.org/O000-0003-1067-727X) Performed the surgeries, conceived and planned the activities that led to the study, critical analysis and approved the final version. All authors read and approved the final manuscript. *ORCID (Open Researcher and Contributor ID) iD).

\section{References}

1. Hueter C. Klinik der Gelenkkrankheiten mit Einschluss der Orthopadie. Leipzig: Verlag Von FCW Vogel; 1871.

2. Nery CAS. Hallux valgus. Rev Bras Ortop. 2001;36(6):183-200.

3. Hamilton GA, Mullins S, Schuberth JM, Rush SM, Ford L. Revision lapidus arthrodesis: rate of union in 17 cases. J Foot Ankle Surg. 2007;46(6):447-50

4. Salomão O. Hallux valgus: etiology and treatment. Rev Bras Ortop. 2005;40(4):147-52.

5. Mansur NSB, de Souza Nery CA. Hypermobility in Hallux Valgus. Foot Ankle Clin. 2020;25(1):1-17.

6. Espinosa N, Wirth $\mathrm{SH}$. Tarsometatarsal arthrodesis for management of unstable first ray and failed bunion surgery. Foot Ankle Clin. 2011;16(1):21-34.

7. Taylor NG, Metcalfe SA. A review of surgical outcomes of the Lapidus procedure for treatment of hallux abductovalgus and degenerative joint disease of the first MCJ. Foot (Edinb). 2008;18(4):206-10.

8. Wai-Chi Wong D, Wang Y, Zhang M, Kam-Lun Leung A. Functional restoration and risk of non-union of the first metatarsocuneiform arthrodesis for hallux valgus: A finite element approach. J Biomech. 2015;48(12):3142-8.

9. Young NJ, Zelen CM. New techniques and alternative fixation for the lapidus arthrodesis. Clin Podiatr Med Surg. 2013;30(3):423-34.

10. Mallette JP, Glenn CL, Glod DJ. The incidence of nonunion after Lapidus arthrodesis using staple fixation. J Foot Ankle Surg. 2014;53(3):303-6.

11. Prissel MA, Hyer CF, Grambart ST, Bussewitz BW, Brigido SA, DiDomenico LA, et al. A Multicenter, Retrospective Study of Early Weightbearing for Modified Lapidus Arthrodesis. J Foot Ankle Surg. 2016;55(2):226-9.

12. Peterson KS, McAlister JE, Hyer CF, Thompson J. Symptomatic Hardware Removal After First Tarsometatarsal Arthrodesis. J Foot Ankle Surg 2016;55(1):55-9.
13. Schmid T, Krause F. The modified Lapidus fusion. Foot Ankle Clin. 2014;19(2):223-33.

14. Klos K, Wilde CH, Lange A, Wagner A, Gras F, Skulev HK, et al. Modified Lapidus arthrodesis with plantar plate and compression screw for treatment of hallux valgus with hypermobility of the first ray: a preliminary report. Foot Ankle Surg. 2013;19(4):239-44.

15. Barp EA, Erickson JG, Smith HL, Almeida K, Millonig K. Evaluation of Fixation Techniques for Metatarsocuneiform Arthrodesis. J Foot Ankle Surg. 2017;56(3):468-73.

16. Blitz NM, Lee T, Williams K, Barkan H, DiDimenico LA. Early weight bearing after modified lapidus arthodesis: a multicenter review of 80 cases. J Foot Ankle Surg. 2010;49(4):357-62.

17. Moore KR, Howell MA, Saltrick KR, Catanzariti AR. Risk Factors Associated With Nonunion After Elective Foot and Ankle Reconstruction: A Case-Control Study. J Foot Ankle Surg. 2017; 56(3):457-62.

18. Klemola T, Leppilahti J, Kalinainen S, Ohtonen P, Ojala R, Savola O. First tarsometatarsal joint derotational arthrodesis--a new operative technique for flexible hallux valgus without touching the first metatarsophalangeal joint. J Foot Ankle Surg. 2014;53(1):22-8.

19. Simons P, Fröber R, Loracher C, Knobe M, Gras F, Hofmann GO, et al. First Tarsometatarsal Arthrodesis: An Anatomic Evaluation of Dorsomedial Versus Plantar Plating. J Foot Ankle Surg. 2015; 54(5):787-92.

20. Li S, Myerson MS. Evolution of Thinking of the Lapidus Procedure and Fixation. Foot Ankle Clin. 2020;25(1):109-26.

21. Conti MS, Patel TJ, Zhu J, Elliott AJ, Conti SF, Ellis SJ. Association of First Metatarsal Pronation Correction With Patient-Reported Outcomes and Recurrence Rates in Hallux Valgus. Foot Ankle Int. 2021;6:10711007211046938 